\title{
Binary neutron star mergers of quark matter based nuclear equations of state
}

\author{
Atul Kedia ${ }^{1, *}$, Grant Mathews ${ }^{1, * *}$, Hee Il Kim² ${ }^{2}$ and In-Saeng Suh ${ }^{3,1}$ \\ ${ }^{1}$ Center for Astrophysics, Department of Physics, University of Notre Dame, Indiana, USA \\ ${ }^{2}$ Center for Quantum Spacetime, Sogang University, Seoul 04107, Korea \\ ${ }^{3}$ Center for Research Computing, University of Notre Dame, Indiana, USA
}

\begin{abstract}
With observations of gravitational wave signals from binary neutron star mergers (BNSM) by LIGO-Virgo-KAGRA (LVK) Collaboration and NICER, the nuclear equation of state (EOS) is becoming increasingly testable by complementary numerical simulations. Numerous simulations currently explore the EOS at different density regimes for the constituent neutron stars specifically narrowing the uncertainty in the sub-nuclear densities. In this paper we summarize the three-dimensional general relativistic-hydrodynamics based simulations of BNSMs for EOSs with a specific emphasis on the quark matter EOS at the highest densities.
\end{abstract}

\section{Introduction}

Neutron stars are ideal locations to probe the properties of matter at the highest density. The microphysics of nuclear interactions in a neutron star are reflected in its large structural features like its mass-radius relation. These interactions also modulate the evolution of neutronstar binary systems. This amplification of subatomic physics makes probing the physics at scales $10^{-14} \mathrm{~m}$ plausible through the observation of neutron star typically of sizes $\sim 10^{4} \mathrm{~m}$. In particular, neutron star binary systems provide a means to analyze the pressure of nuclear matter at all domains of nuclear densities. The first detection of gravitational waves from the binary neutron star merger GW170817 by the LIGO-Virgo Collaboration and the pulsar PSR J0740 by NICER have provided new insights into the form and structure of dense neutronstar matter. [1,2] The gravitational wave signal detected thus is dependent upon the tidal distortion of the neutron stars as they approach merger. The LIGO analysis $[1,3]$ of the tidal polarizability $[4,5]$ deduced from post-Newtonian dynamics implies that the radius of the stars of $1.4 \mathrm{M}_{\odot}$ is in the range $10.5 \mathrm{~km} \leq R \leq 13.3 \mathrm{~km}$, which has placed tight constrains on the equation of state (EOS) for nuclear matter as the stars approach merger.

Here we summarize the literature in the regime of quantum chromodynamics (QCD) attained in the core of the short-lived massive neutron star formed after the merger of a neutron star binary, and the potential locations to look at for signs from the QCD regime. The reason to be excited about this, particularly now, is that the detection of the gravitational radiation during the postmerger could be used as a sensitive probe of both the order of the quarkhadron phase transition and the properties of matter in the non-perturbative regime of QCD.

\footnotetext{
*e-mail: atulkedia93@gmail.com

**e-mail: gmathews@nd.edu
} 
The prospect of the postmerger evolution being used to explore EOS issues has been proposed for some time (e.g. [6]). There have also been several investigations into the effects of the formation of quark matter in the BNSM. [7-10]. For the most part these studies have considered effects of a first-order phase transition and the formation of a mixed quark-hadron phase. However, a first-order transition can soften the EOS and hasten the formation of the imminent black hole.

The postmerger gravitational wave strain lies in a frequency range of $2 \mathrm{kHz}$ to 5 $\mathrm{kHz}$ and as a result the strength for a binary at $50 \mathrm{Mpc}$ is not easily accessible to aLIGO/aVirgo/KAGRA. However, the third generation GW observatories, such as the Einstein Telescope and the Cosmic Explorer, will have enhanced sensitivities in this frequency range and will be capable of observing the postmerger evolution of a BNSM. Furthermore, there is a suggestion in the literature (see Refs. [11, 12]) of post merger energy output in gravitational radiation from the GW170817 event that appears to be an extended ringdown (see however Ref. [13].) In Ref. [12] it was hypothesized that such extended emission might result from spin down of a magnetar.

\section{Dense matter Equation of State}

To describe the evolution of matter in a closed form, the hydrodynamics equations require an additional constraint that relates the various state variables of the matter, i.e. pressure, density, electron fraction, chemical potentials, etc. in a neutron star. [14, 15] Constraints on the low density (sub-nuclear) equation of state (EOS) have been placed by aLIGO based upon the tidal polarizability deduced from the chirp associated with event GW170817. [1, 3] However the EOS at higher densities attained during a binary neutron star merger must also include the consequences of a transition between hadronic matter and quark matter. As the merged system collapses to a black hole it unavoidably encounters certain high dense phases of matter, particularly the transition to quark matter.

It is worth noting that as the baryon density and chemical potential increase the QCD strong coupling constant $\alpha_{s}$ approaches unity and a nonperturbative approach to QCD is imperative. In particular, there is rich physics in this region of the quark-matter phase diagram including the generation of constituent quark masses, due to chiral symmetry breaking [16], and quark pairing leading to color superconductivity [17]. The evolution of these effects until the asymptotic regime must be described during the collapse.

The transition from hadronic matter to quark matter is not yet fully understood. [18] However, people have proposed many interesting models to describe the transition from hadronic matter to quark matter at densities between $2-5 \rho_{\text {nuc }}$. $[19,20]$ Considering strongly interacting quark states for the crossover density region, people could have made the EOSs satisfying 2 times solar mass observational bound. [21] Also, many models having the first order phase transition features have been proposed to describe the transition.

\section{Gravitational Waves and Power Spectral Density}

In addition to $f_{\text {max }}$ which could be obtained from the instantaneous change of the phase of $h$ at the chirp, the power spectral density (PSD) of the strain, poses to reveal further features about the star's EOS. Refs. [10,22] shows the presence of high frequency spectral features in the $f_{1}, f_{2}$ and $f_{3}$ (as defined in Ref. [22]) modes for binaries with different EOS description. The positioning and strength of the frequencies depends on the nature of the EOS. One of the important aspects about the power spectra of the mergers is that the strength of the PSD at $2 \mathrm{kHz}$ to $5 \mathrm{kHz}$ frequencies is weak enough that the current LVK detectors are unable to 
discern them from the noise. It is also pointed out that the third generation gravitational wave interferometers, the Einstein Telescope and the Cosmic Explorer, would have higher sensitivities at these frequencies making them more likely to resolve the $f$-modes. Further, Ref. [22] notes the occurrence of 'quasiuniversal' relations relating $f$-modes with compactness and the maximum frequency of chirp with tidal deformability of a single stable NS for the given EOS. These relations can be explained partially by the stiffness of the equation of state, softer EOSs leading to higher $f$-modes. Recent studies of the BNSM with the phase transition have examined whether the features of the phase transition could be imprinted in the f-mode frequencies, for example, the shift of the peak frequencies. [8, 23] We are currently investigating the spectral features of the BNSM with a specific crossover EOS and have found some interesting postmerger behaviour showing elongated duration owning to the stiffened EOS. [18, 19, 24]

\section{Conclusion}

We briefly summarize recent studies of BNSM with quark matter EOSs. An observation of the postmerger and the collapse in a BNSM could be used to determine the nature of the phase transition and the physics at the crossover densities. The next generation GW detectors will probe and unveil the physics of this dense matter physics with their highly improved power of detectabilities at higher $\mathrm{GW}$ frequencies.

Finally, noting a caveat that there have been no studies fully considering aspects such as magnetohydrodynamics and neutrino transport for the simulations, however, we expect that essential features of the non-perturbative characters of the QCD would be revealed and confirmed without needing to take these considerations into account.

\section{References}

[1] B.P. Abbott et al. (LIGO Scientific Collaboration and Virgo Collaboration), Phys. Rev. Lett. 119, 161101 (2017).

[2] M. C. Miller et al 2021, The Astrophysical Journal Letters, 918 L28

[3] B. P. Abbott, et al. "Tests of general relativity with GW170817." Phys. Rev. Lett. 123.1 (2019): 011102.

[4] T. Damour, M. Soffel, and C. Xu, Phys. Rev. D 45, 1017 (1992).

[5] E. E. Flanagan and T. Hinderer, Phys. Rev. D 77, 021502 (2008).

[6] A. Bauswein, H.-T. Janka, K. Hebeler, and A. Schwenk, Phys. Rev. D 86, 063001 (2012).

[7] E. R. Most, L. Jens Papenfort, V. Dexheimer, M. Hanauske, H. Stoecker, and L. Rezzolla, On the de-confinement phase transition in neutron-star mergers, Eur. Phys. J. A 56, 59 (2020)

[8] L. R. Weih, M. Hanauske, and L. Rezzolla, Postmerger Gravitational-Wave Signatures of Phase Transitions in Binary Mergers, Phys. Rev. Lett. 124, 171103 (2020).

[9] S. L. Liebling, C. Palenzuela, and L. Lehner, Effects of High Density Phase Transitions on Neutron Star Dynamics, Class. Quant. Grav. 38, 115007 (2021)

[10] A. Prakash, D. Radice, D. Logoteta, et al., arXiv:2106.07885 (2021)

[11] H. M. P van Putten and D. V. Massimo, MNRAS, 482, L46 (2019).

[12] H. M. P van Putten, D. V. Massimo, and A. Levinson, Astrophys. J. Lettt, 876, L2 (2019).

[13] M. Oliver, D. Keitel, A. Miller, et al. MNRAS, 485, 843 (2019).

[14] D. Radice, A. Perego, F. Zappa, and S. Bernuzzi Ap. J. Lett. 852.2 (2018): L29. 
[15] J. Olson, et al., arXiv:1612.08992 (2016).

[16] Hatsuda, T., and Kunihiro, T. 1994, PhR, 247, 221

[17] M. G. Alford, A. Schmitt, K. Rajagopal, and T. Schäfer, 2008, RvMP, 80, 1455.

[18] Baym, G., Hatsuda, T., Kojo, T., et al. 2018, RPPh, 81, 056902

[19] G. Baym, S. Furusawa, T. Hatsuda, T. Kojo, H. Togashi, ApJ, 885, 42 (2019)

[20] E. Annala, E., T. Gorda, A. Kurkela, et al., Nature Physics, (2020), 16(9), 907-910.

[21] P. B. Demorest, T. Pennucci, S. M. Ransom, et al. , Nature (London) 467, 1081 (2010).

[22] K. Takami, L. Rezzolla, and L. Baiotti, Physical Review D 91.6 (2015): 064001

[23] S. Blacker, NUF Bastian, A. Bauswein, et al., Physical Review D 102.12 (2020): 123023.

[24] A. Kedia, H.I. Kim, I.S. Suh, G. Mathews, (2022) in preparation. 